\title{
Simple model for the spherically- and system-averaged pair density: Results for two-electron atoms
}

\author{
Paola Gori-Giorgi and Andreas Savin \\ Laboratoire de Chimie Théorique, CNRS, Université Pierre et Marie Curie, 4 Place Jussieu, F-75252 Paris, France
}

(Dated: November 19, 2018)

\begin{abstract}
As shown by Overhauser and others, accurate pair densities for the uniform electron gas may be found by solving a two-electron scattering problem with an effective screened electron-electron repulsion. In this work we explore the extension of this approach to nonuniform systems, and we discuss its potential for density functional theory. For the spherically- and system-averaged pair density of two-electron atoms we obtain very accurate short-range properties, including, for nuclear charge $Z \geq 2$, "on-top" values (zero electron-electron distance) essentially indistinguishable from those coming from precise variational wavefunctions. By means of a nonlinear adiabatic connection that separates long- and short-range effects, we also obtain Kohn-Sham correlation energies whose error is less than $4 \mathrm{mHartree}$, again for $Z \geq 2$, and short-range-only correlation energies whose accuracy is one order of magnitude better.
\end{abstract}

\section{INTRODUCTION AND SUMMARY OF RESULTS}

Density Functional Theory (DFT) [1, 2, 3] is nowadays the most widely used method for electronic structure calculations, in both condensed matter physics and quantum chemistry, thanks to a combination of low computational cost and reasonable accuracy.

In the application of this theory within the Kohn-Sham (KS) formalism [4], one deals with a model system (the KS system) of $N$ noninteracting electrons in a local potential $v_{\mathrm{KS}}(\mathbf{r})$ that forces them to yield the same density $n(\mathbf{r})$ of the physical system. The energy of the physical system is then obtained from that of the KS system via a functional of the density, whose only term not explicitly known is the exchange-correlation energy $E_{x c}[n]$. Correspondingly, in the local potential $v_{\mathrm{KS}}(\mathbf{r})$ there is an unknown term, $v_{x c}(\mathbf{r})=\delta E_{x c}[n] / \delta n(\mathbf{r})$.

The success of KS DFT is mostly due to the fact that even simple physical approximations of $E_{x c}[n]$, like the local density approximation (LDA) [4], already give acceptable results for many purposes. This spurred fundamental research in the field, and led to a wealth of more and more sophisticated exchange-correlation functionals 2, 3, 5], and to the development of different approaches to DFT [6, 7].

Recently, in the search for accurate $E_{x c}[n]$, the focus of a large part of the DFT community has shifted from seeking explicit functionals of the density like the generalized gradient approximations (GGA) [8], to implicit functionals, tipically using the Kohn-Sham orbital kinetic energy density 9] or the Kohn-Sham orbitals (see, e.g., [3, 10, 11]). The so-called "third generation" of exchange-correlation functionals is based on the exact exchange of the noninteracting (KS) system, simply obtained by putting in the formal expression for the Hartee-Fock exchange the Kohn-Sham orbitals $\varphi_{i \sigma}(\mathbf{r})$. Such expression corresponds to an implicit functional of the density, $E_{x}[n]=E_{x}\left[\left\{\varphi_{i \sigma}[n]\right\}\right]$. The local potential $v_{x}(\mathbf{r})=\delta E_{x}[n] / \delta n(\mathbf{r})$ that generates the orbitals $\varphi_{i \sigma}(\mathbf{r})[n]$ can be obtained via the optimized effective po- tential method (OEP) [12.

In this broad context, sketchily summarized here, we propose a simplified method to build the "bridge" between the physical and the KS system, or, more generally, with a reference model system of partially interacting electrons. We focus on a quantity which is known to play a crucial role in DFT and has an intuitive physical meaning, the spherically and system-averaged electronic pair density $f\left(r_{12}\right)$ (also known in chemistry as spherical average of the intracule density, see e.g. 13, 14, 15, 16], and especially 17,18$]$ ). Given the spin-resolved diagonal of the two-body reduced density matrix,

$$
\gamma_{\sigma_{1} \sigma_{2}}^{(2)}\left(\mathbf{r}_{1}, \mathbf{r}_{2}\right)=\sum_{\sigma_{3} \ldots \sigma_{N}} \int\left|\Psi\left(\mathbf{r}_{1} \sigma_{1}, \ldots, \mathbf{r}_{N} \sigma_{N}\right)\right|^{2} d \mathbf{r}_{3} \ldots d \mathbf{r}_{N}
$$

we define the spin-summed pair density $n_{2}\left(\mathbf{r}_{1}, \mathbf{r}_{2}\right)$,

$$
n_{2}\left(\mathbf{r}_{1}, \mathbf{r}_{2}\right)=\frac{N(N-1)}{2} \sum_{\sigma_{1} \sigma_{2}} \gamma_{\sigma_{1} \sigma_{2}}^{(2)}\left(\mathbf{r}_{1}, \mathbf{r}_{2}\right)
$$

and we integrate it over all variables but $r_{12}=\left|\mathbf{r}_{2}-\mathbf{r}_{1}\right|$ by switching, e.g., to center-of-mass coordinates, $\mathbf{R}=$ $\frac{1}{2}\left(\mathbf{r}_{1}+\mathbf{r}_{2}\right), \mathbf{r}_{12}=\mathbf{r}_{2}-\mathbf{r}_{1}$

$$
f\left(r_{12}\right)=\int d \mathbf{R} \frac{d \Omega_{\mathbf{r}_{12}}}{4 \pi} n_{2}\left(\mathbf{R}-\frac{\mathbf{r}_{12}}{2}, \mathbf{R}+\frac{\mathbf{r}_{12}}{2}\right) .
$$

The function $f\left(r_{12}\right)$ times the volume element $4 \pi r_{12}^{2} d r_{12}$ is proportional to the probability density for the particleparticle distance in a system of $N$ electrons in the state $\Psi$, and is normalized to the number of electron pairs, $N(N-1) / 2$. This quantity fully determines the expectation value of the electronic Coulomb repulsion (in Hartree atomic units used throughout),

$$
\left\langle V_{e e}\right\rangle \equiv\left\langle\Psi\left|V_{e e}\right| \Psi\right\rangle=\int_{0}^{\infty} 4 \pi r_{12}^{2} \frac{f\left(r_{12}\right)}{r_{12}} d r_{12},
$$

and is a measurable quantity, being essentially the Fourier transform of the electronic static structure factor [19]. By construction, the one-electron density $n(\mathbf{r})$ is 

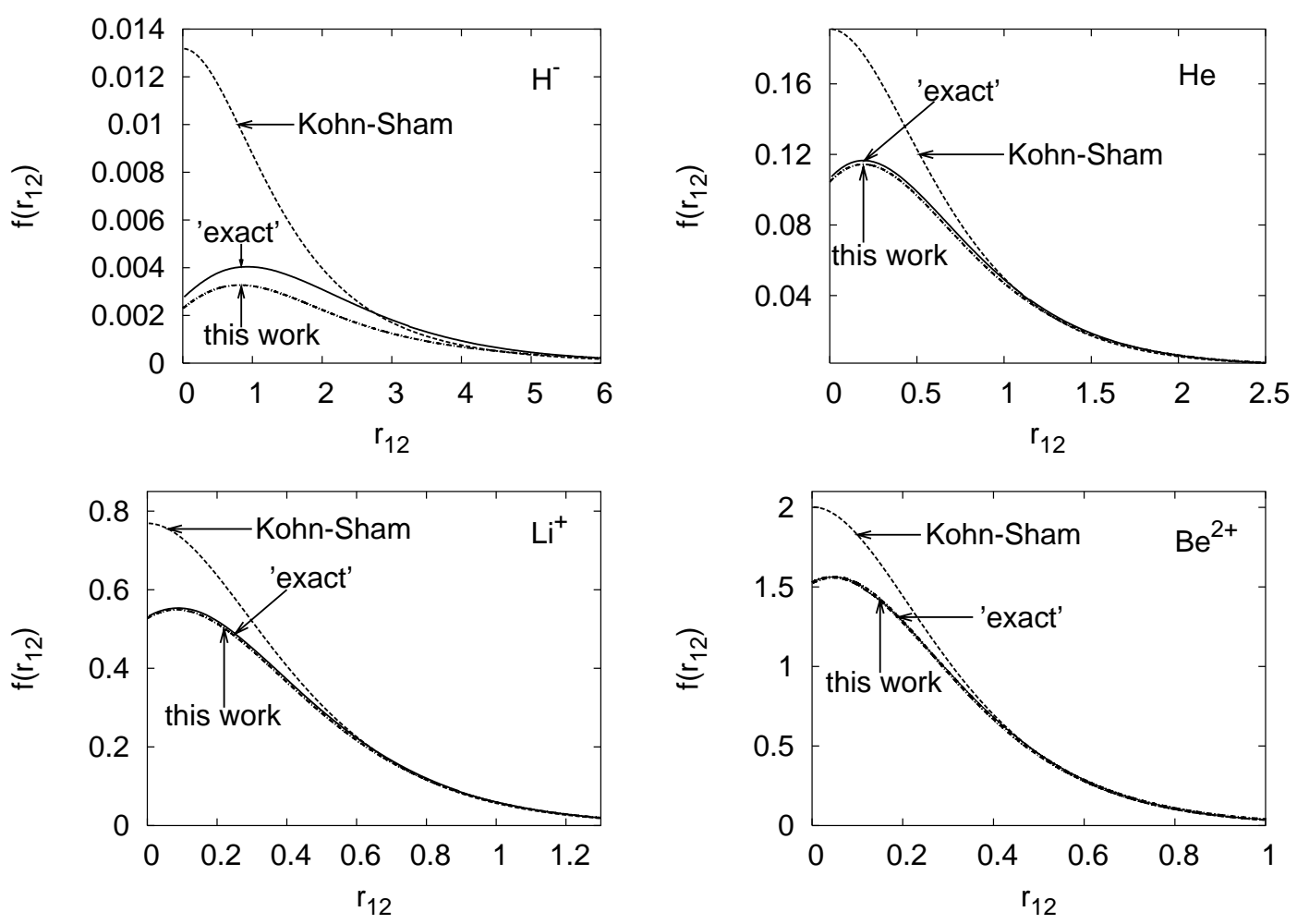

FIG. 1: Spherically and system-averaged pair densities for two-electron atoms: 'exact' results [40] are compared with the values obtained for the Kohn-Sham system and with the present approach, which is designed to get realistic $f\left(r_{12}\right)$ starting from the Kohn-Sham ones.

the same in the KS and in the physical system, whereas $f\left(r_{12}\right)$ will be different in the two cases, as shown, e.g., in Fig. 10 for some two-electron atoms. In the physical system $f\left(r_{12}\right)$ has a much lower "on-top" value $f\left(r_{12}=0\right)$ than in the KS system, and it has a cusp 20], as expected from the fact that the electrons repel each other via the Coulomb interaction. Roughly speaking, in the classic DFT approach to correlation, the difference in energy arising when we evaluate the r.h.s. of Eq. (4) with the two $f\left(r_{12}\right)$, the physical and the KS, is what one tries to describe with a universal functional of the density 21]. Here we follow a different approach: we try to build realistic $f\left(r_{12}\right)$ from a set of simple radial equations, to be solved for each system, and eventually coupled to a DFT calculation.

Our approach is inspired by the seminal work of Overhauser [22] and its subsequent extension [23], in which the function $f\left(r_{12}\right)$ for the uniform electron gas is obtained from a set of geminals, solutions of a radial Schrödinger equation with an effective electron-electron $(e-e)$ potential. Simple approximations for such effective $e-e$ potential give indeed accurate results at all relevant densities 23, 24, 25]. Here we try to generalize this approach to systems of nonuniform density to get accurate $f\left(r_{12}\right)$. The main goal of the present work is understanding whether the method is promising, and whether it is worth developing and refining it. To this purpose, we define the formalism (Sec. III), we give a physically-motivated prescription for the effective $e$-e potential (Sec. IIII), and we test it on the simple but not trivial case of two-electron atoms (Sec. IV). The prescription for the effective $e-e$ potential used here is not very sophisticated. Improvements along the lines of what has been done for the uniform electron gas 23, 24, 25] will be the subject of future work. Yet, even at this simple first stage of the theory we already obtain rather accurate results, especially for the short-range part of $f\left(r_{12}\right)$ (see Fig. [1 and Table [). In Sec. $\nabla$ we show that with the present approach we can also recover the difference in kinetic energy between the physical and the KS system. Finally, Sec. [VI is devoted to conclusions, perspectives and open questions.

\section{FORMALISM}

In addition to the work on the "Overhauser model" 22, 23, 24], the approach described here takes advantage of inspiring papers on the possibility of constructing a pairdensity functional theory [26, 27, 28, 29], and a localdensity-of-states functional theory [30].

Our starting point is a constrained search over $\frac{1}{2} N(N-$ 1) "effective" orthonormal geminals $\psi_{i}\left(r_{12}\right)$ that minimize the electron-electron relative kinetic energy $T_{12}=$ $-\nabla_{r_{12}}^{2}$ (the reduced mass for the relative motion is $1 / 2$ ) 
and yield the exact $f, \sum_{i}\left|\psi_{i}\left(r_{12}\right)\right|^{2}=f\left(r_{12}\right)$,

$$
\min _{\left\{\psi_{i}\right\} \rightarrow f} \sum_{i}\left\langle\psi_{i}\left|-\nabla_{r_{12}}^{2}\right| \psi_{i}\right\rangle,
$$

thus leading to a set of radial equations formally similar to the KS ones,

$$
\begin{aligned}
& {\left[-\nabla_{r_{12}}^{2}+v_{\text {eff }}\left(r_{12}\right)\right] \psi_{i}\left(r_{12}\right)=\epsilon_{i} \psi_{i}\left(r_{12}\right)} \\
& \sum_{i=1}^{N(N-1) / 2}\left|\psi_{i}\left(r_{12}\right)\right|^{2}=f\left(r_{12}\right) .
\end{aligned}
$$

These equations imply that an expansion in spherical harmonics of $f\left(r_{12}\right)$ has been done, so that the kinetic energy operator also contains the usual $\ell(\ell+1) / r^{2}$ term. To fully define these equations we need a rule for the occupancy of the effective geminals. In analogy with what has been done for the uniform electron gas [23, 24], we can assign spin degeneracy 1 to even-angular-momentum states (singlet) and spin degeneracy 3 to odd-angularmomentum states (triplet), up to $N(N-1) / 2$ occupied states. More generally, for open shell systems it could be better to develop the formalism for the spin-resolved quantities, starting from Eq. (11). This will be investigated in future work.

The effective electron-electron potential $v_{\text {eff }}\left(r_{12}\right)$ of Eq. (6) is the Lagrange parameter for $f\left(r_{12}\right)$, and is a functional of $f$ itself and of the electron-nucleus external potential $V_{n e}$ (or, equivalently, of the density $n(\mathbf{r})$ ). To see this, we can rewrite our Eqs. (6)-(7) in terms of a minimization of the total energy in two steps, using the constrained search formalism [31, 32] for the ground state energy $E=\min _{\Psi}\left\langle\Psi\left|T+V_{e e}+V_{n e}\right| \Psi\right\rangle$,

$$
\begin{aligned}
E= & \min _{f} \min _{\Psi \rightarrow f}\left\{\min _{\left\{\psi_{i}\right\} \rightarrow f} \sum_{i}\left\langle\psi_{i}\left|-\nabla_{r_{12}}^{2}\right| \psi_{i}\right\rangle+\int \frac{f}{r_{12}} d \mathbf{r}_{12}\right. \\
& \left.+\left\langle\Psi\left|T+V_{n e}\right| \Psi\right\rangle-\min _{\left\{\psi_{i}\right\} \rightarrow f} \sum_{i}\left\langle\psi_{i}\left|-\nabla_{r_{12}}^{2}\right| \psi_{i}\right\rangle\right\} . \text { (8) }
\end{aligned}
$$

Defining the kinetic and external-potential functional as

$$
\begin{array}{r}
F_{\mathrm{KE}}\left[f ; V_{n e}\right]= \\
\min _{\Psi \rightarrow f}\left\langle\Psi\left|T+V_{n e}\right| \Psi\right\rangle-\min _{\left\{\psi_{i}\right\} \rightarrow f} \sum_{i}\left\langle\psi_{i}\left|-\nabla_{r_{12}}^{2}\right| \psi_{i}\right\rangle,
\end{array}
$$

we can rewrite

$$
\begin{aligned}
E= & \min _{f}\left\{\min _{\left\{\psi_{i}\right\} \rightarrow f} \sum_{i}\left\langle\psi_{i}\left|-\nabla_{r_{12}}^{2}\right| \psi_{i}\right\rangle+\int \frac{f}{r_{12}} d \mathbf{r}_{12}\right. \\
& \left.+F_{\mathrm{KE}}\left[f ; V_{n e}\right]\right\} .
\end{aligned}
$$

Searching this minimum by directly varying the $\psi_{i}$ (with given, fixed, $V_{n e}$ ) leads to Eqs. (66)-(77) with the identification

$$
v_{\mathrm{eff}}\left(r_{12}\right)=\frac{1}{r_{12}}+\frac{\delta F_{\mathrm{KE}}\left[f ; V_{n e}\right]}{\delta f\left(r_{12}\right)} .
$$

Thus, in principle we could recover the whole groundstate energy via the (unknown) system-dependent functional $F_{\mathrm{KE}}\left[f ; V_{n e}\right]$. In practice, it seems much more feasible to combine Eqs. (6)-(7) with a DFT calculation, that yields the complementary information (the density, and thus $\left\langle\Psi\left|V_{n e}\right| \Psi\right\rangle$ ). The steps of Eqs. (8)-(11) can be repeated for arbitrary electron-electron interaction and external one-body potential. In particular, we can set $V_{e e}^{\lambda}=\lambda V_{e e}$ and $V_{n e}=V^{\lambda}$, where $V^{\lambda}$ is an external potential that keeps the density equal to the one of the physical system. One could thus obtain $f^{\lambda}$ at each coupling strength $\lambda$ between 0 and 1 from Eqs. (6)-(7) with a suitable $v_{\text {eff }}^{\lambda}$. The correlation energy of KS theory is then simply given by $[6,33,34$ ]

$$
E_{c}[n]=\int_{0}^{1} d \lambda \int d \mathbf{r}_{12} \frac{f^{\lambda}\left(r_{12}\right)-f^{\lambda=0}\left(r_{12}\right)}{r_{12}} .
$$

Alternatively, this procedure (usually called adiabatic connection [34]) can be performed along a nonlinear path, e.g., by setting [6, 35, 36, 37] $v_{e e}^{\lambda}=\operatorname{erf}(\lambda r) / r$, where $\operatorname{erf}(x)$ is the error function (see Sec. $\nabla)$. Eventually, the two sets of equations, KS and (6)-(17) plus (12), could be solved together self-consistently. This last issue is discussed in Sec. VI Notice that if we combine Eqs. (6), (17) and (12) with a DFT calculation, we only need to approximate the potential $v_{\text {eff }}^{\lambda}\left(r_{12}\right)$ and not the whole functional $F_{\mathrm{KE}}$ since the remaining information is provided by DFT.

It is also worth to stress at this point that there is no wavefunction behind our Eqs. (6)-(17): the effective geminals $\psi_{i}$ are defined via Eq. (5), and by specifying their occupancy (e.g., triplet and singlet). A bosonic version of the theory, in which only one geminal (proportional to $\sqrt{f\left(r_{12}\right)}$ ) is occupied can also be considered [29, [38]. In this work we only focus on two-electron systems for which the two choices are equivalent. A careful comparison of performances of the "fermion-like" and of the "boson-like" occupancy in the uniform electron gas is the subject of current investigations [39].

As for KS DFT, the formalism just described can be useful only if simple approximations for $v_{\text {eff }}\left(r_{12}\right)$ yield accurate results. This is what we start to check in the rest of this paper. First, we construct a physically-motivated $v_{\text {eff }}$ for two-electron atoms for the fully-interacting system, and we compare our results with "exact" ones [40]. Then, we generalize our construction to build $v_{\text {eff }}$ along the adiabatic connection, and we calculate the KS correlation energy.

\section{EFFECTIVE ELECTRON-ELECTRON POTENTIAL: THE OVERHAUSER MODEL}

For the interacting electron gas of uniform density $n$, Overhauser 22] proposed a simple and reasonable effective potential $v_{\text {eff }}\left(r_{12}\right)$ : he took the sphere of volume $n^{-1}$ around a given electron as the boundary within which the other electrons are excluded, due to exchange and 
correlation effects. In the standard uniform-electron-gas model, a rigid positively-charged background maintains the electrical neutrality. Thus the exclusion region (or "hole") around a given electron, modeled with a sphere of radius $r_{s}=(4 \pi n / 3)^{-1 / 3}$, uncovers the background of positive charge, leading to an effective screened Coulomb potential with screening length $r_{s}$,

$$
v_{\mathrm{eff}}^{O v}\left(r_{12} ; r_{s}\right)=\frac{1}{r_{12}}-\int_{|\mathbf{r}| \leq r_{s}} \frac{n}{\left|\mathbf{r}-\mathbf{r}_{12}\right|} d \mathbf{r},
$$

equal to

$$
\begin{aligned}
& v_{\text {eff }}^{O v}\left(r_{12} ; r_{s}\right)=\frac{1}{r_{12}}+\frac{r_{12}^{2}}{2 r_{s}^{3}}-\frac{3}{2 r_{s}} \quad r_{12} \leq r_{s} \\
& v_{\text {eff }}^{O v}\left(r_{12} ; r_{s}\right)=\quad 0 \quad r_{12}>r_{s} \text {. }
\end{aligned}
$$

Equations (6)-(7), combined with the Overhauser effective potential of Eq. (14) gave extremely accurate results for the short-range part $\left(r_{12} \leq r_{s}\right)$ of the function $f\left(r_{12}\right)$ in the uniform electron gas at all relevant densities [23]. A more sophisticated effective potential, based on a selfconsistent Hartree approximation, extended such accuracy to the long-range part of $f\left(r_{12}\right)$ at metallic densities [24]. Other approximate $v_{\text {eff }}\left(r_{12}\right)$ for the uniform electron gas have also been proposed [25], and exact properties have been derived [41].

To produce realistic $f\left(r_{12}\right)$ for nonuniform systems from Eqs. (6)-(7), here we generalize the original idea of Overhauser 22, 23] to two-electron atoms, and show that it gives rather accurate results, especially for the short-range part of $f\left(r_{12}\right)$. We start from the effective potential $v_{\text {eff }}^{(0)}\left(r_{12}\right)$ that generates $f_{\mathrm{KS}}\left(r_{12}\right)$, the sphericallyand system averaged pair density of the Kohn-Sham system. In the special case of a spin-compensated twoelectron system, the $\mathrm{KS}$ wavefunction is simply equal to $\frac{1}{2} \sqrt{n\left(\mathbf{r}_{1}\right)} \sqrt{n\left(\mathbf{r}_{2}\right)}$. Because, at this first stage, we are interested in testing our method as a "bridge" between the KS and the real system, here we use the "exact" Kohn-Sham system. We thus take accurate one-electron densities [40], and construct $f_{\mathrm{KS}}\left(r_{12}\right)$,

$$
f_{\mathrm{KS}}\left(r_{12}\right)=\frac{1}{4} \int n\left(\mathbf{R}-\frac{\mathbf{r}_{12}}{2}\right) n\left(\mathbf{R}+\frac{\mathbf{r}_{12}}{2}\right) d \mathbf{R} \frac{d \Omega_{\mathbf{r}_{12}}}{4 \pi},
$$

and the corresponding "exact" potential $v_{\mathrm{eff}}^{(0)}\left(r_{12}\right)$, that can be calculated by inverting Eqs. (6)-(7),

$$
v_{\mathrm{eff}}^{(0)}=\frac{\nabla^{2} \sqrt{f_{\mathrm{KS}}}}{\sqrt{f_{\mathrm{KS}}}}+\text { const. }
$$

For systems with more than two electrons, the potential $v_{\text {eff }}^{(0)}$ could be calculated, e.g., with the methods of Refs. [42, 43]. In practice, it would be much more efficient to build approximations also for $v_{\text {eff }}^{(0)}$ (see Sec. VI). Examples of functions $f_{\mathrm{KS}}$ for nuclear charges $Z=1,2,3,4$ are given in Fig. 1] they have a maximum at $r_{12}=0$, as expected in a system of two non-interacting electrons with antiparallel spins in a confining one-body external

\begin{tabular}{llllll}
\hline \hline & $\mathrm{H}^{-}$ & $\mathrm{He}$ & $\mathrm{Li}^{+}$ & $\mathrm{Be}^{2+}$ & $\mathrm{Ne}^{8+}$ \\
\hline $\bar{r}_{s}$ & 2.1 & 0.86 & 0.54 & 0.39 & 0.15 \\
& & & & & \\
$f(0)$ & 0.0021 & 0.104 & 0.528 & 1.526 & 32.6 \\
"exact" & 0.0027 & 0.106 & 0.534 & 1.523 & 32.7 \\
LDA & 0.0047 & 0.119 & 0.563 & 1.587 & 33.0 \\
& & & & & \\
$r_{12}^{\max }$ & 0.835 & 0.193 & 0.083 & 0.0465 & 0.0074 \\
"exact" & 0.927 & 0.194 & 0.083 & 0.0465 & 0.0074 \\
& & & & & \\
$f\left(r_{12}^{\max }\right)$ & 0.0031 & 0.114 & 0.55 & 1.56 & 32.74 \\
"exact" & 0.0040 & 0.117 & 0.56 & 1.56 & 32.74 \\
& & & & & \\
$\left\langle V_{e e}\right\rangle-\left\langle V_{e e}\right\rangle_{K S}$ & -0.12 & -0.097 & -0.10 & -0.10 & -0.10 \\
"exact" & -0.07 & -0.078 & -0.082 & -0.089 & -0.09 \\
\hline \hline
\end{tabular}

TABLE I: Our results for the function $f\left(r_{12}\right)$ for two-electron atoms (first line for each property) compared with the corresponding "exact" quantities [40]. In the first line of the table we report the average $\bar{r}_{s}$ as defined by Eqs. (17) and (19). For the "on-top" value $f(0)$ we also show the LDA result (with $f(0)$ for the uniform electron gas from Ref. [23]). All values are in Hartree atomic units.

potential. When the interaction is turned on, the average distance between the two electrons increases, with the constraint that $n(\mathbf{r})$ is kept fixed. We can thus imagine that, with respect to the Kohn-Sham system, in the physical system the Coulomb repulsion between the electrons creates, on average, a screening "hole" around the reference electron of volume $(\bar{n})^{-1}$, where $\bar{n}$ is an average density (i.e., $n(\mathbf{r})$ integrated over the wavefunction),

$$
\bar{n}=\frac{1}{N} \int d \mathbf{r} n(\mathbf{r})^{2} .
$$

An approximate $v_{\text {eff }}\left(r_{12}\right)$ could thus be simply constructed as

$$
v_{\text {eff }}\left(r_{12}\right) \approx v_{\text {eff }}^{(0)}\left(r_{12}\right)+v_{\text {eff }}^{O v}\left(r_{12} ; \bar{r}_{s}\right)
$$

with an average $\bar{r}_{s}$ in $v_{\text {eff }}^{O v}$ of Eq. (14),

$$
\bar{r}_{s}=\left(\frac{4 \pi}{3} \bar{n}\right)^{-1 / 3} \text {. }
$$

The Overhauser-like potential $v_{\text {eff }}^{O v}\left(r_{12} ; \bar{r}_{s}\right)$ is thus a correlation potential to be added to the one that generates $f_{\mathrm{KS}}$. It describes the correlation between pairs of electrons due to Coulomb interaction, and keeps the information on the one-electron density in an approximate way, via the average $\bar{n}$ of Eq. (17). Of course, for more complicated systems we expect to need a more sophisticated construction for $\bar{r}_{s}$.

\section{RESULTS}

We have inserted the potential of Eq. (18) into Eqs. (6)(7), and solved them for several two-electron atoms. Our results are shown in Fig. 11 and summarized in Table [] 
We see that the simple effective potential of Eq. (18) gives already reasonable results for $Z=1$ and 2 , and that the accuracy of the results increases with $Z$ (as the system becomes less and less correlated). The "on-top" value $f(0)$ is essentially exact for $Z \geq 2$, and is much better than the LDA estimate (normally regarded as accurate) for all $Z$. This feature is appealing, since the on-top value plays an important role in DFT [44], and accurate $f(0)$ are not easy to obtain from ab initio methods (see, e.g., Ref. [45] and references therein). The term $1 / r_{12}$ in the effective potential ensures that the calculated $f\left(r_{12}\right)$ satisfies the exact cusp condition $f^{\prime}(0)=f(0)$. Table \also shows that the position $r_{12}^{\max }$ and the height $f\left(r_{12}^{\max }\right)$ of the maximum of $f$ is very well predicted by the present approach. The presence of this maximum is essentially due to the combined effect of the Coulomb repulsion between the electrons and the confining external potential.

In Fig. 2 we consider $\mathrm{He}$ and $\mathrm{Ne}^{8+}$, and we compare the correlated part of our $f, f_{c}=f-f_{\mathrm{KS}}$, with the "exact" result [40] and with the corresponding quantity calculated within LDA, i.e.,

$$
f_{c}^{\mathrm{LDA}}\left(r_{12}\right)=\frac{1}{2} \int n(\mathbf{r})^{2} g_{c}\left(r_{12} ; n(\mathbf{r})\right) d \mathbf{r},
$$

where $g_{c}$ is the pair-correlation function of the uniform electron gas at full coupling strength, taken from Ref. [46]. (For an extended system of uniform density $n$, we have $g_{c}=2 f_{c} / n N$.) Figure 3 shows the same quantities multiplied by $4 \pi r_{12}$, i.e. the integrand of Eq. (4) for the correlation part of $\left\langle V_{e e}\right\rangle$ : the area under each curve gives $\left\langle V_{e e}\right\rangle-\left\langle V_{e e}\right\rangle_{K S}$. In the last line of Table \we report quantitative results for $\left\langle V_{e e}\right\rangle-\left\langle V_{e e}\right\rangle_{K S}$. This quantity is less accurate than the short-range properties, but it is still encouraging. Moreover, it saturates for large $Z$ as in the exact case.

\section{ADIABATIC CONNECTION AND CORRELATION ENERGY}

For the calculation of the energy of the physical system, in addition to $V_{c}[n]=\left\langle V_{e e}\right\rangle-\left\langle V_{e e}\right\rangle_{K S}$, one needs to know the kinetic-energy difference, $T_{c}[n]=\langle T\rangle-\langle T\rangle_{K S}$, that can be obtained via the adiabatic connection formalism 6, 33, 34]. By varying a parameter $\lambda$, the interaction $v_{e e}^{\lambda}\left(r_{12}\right)$ between the electrons is switched on continuously from zero to $1 / r_{12}$, while the density is kept fixed by an external one-body potential $V^{\lambda}$. If $v_{e e}^{\lambda=0}=0$ and $v_{e e}^{\lambda=a}=1 / r_{12}$, the KS correlation energy $E_{c}[n]=T_{c}[n]+V_{c}[n]$ is given by [6, $\left.\underline{34}\right]$

$$
E_{c}[n]=\int_{0}^{a} d \lambda \int_{0}^{\infty} d r_{12} 4 \pi r_{12}^{2} f_{c}^{\lambda}\left(r_{12}\right) \frac{\partial v_{e e}^{\lambda}\left(r_{12}\right)}{\partial \lambda}
$$

where $f_{c}^{\lambda}=f^{\lambda}-f_{\mathrm{KS}}$.

Usually, the adiabatic connection is performed along a linear "path" 11, 33], by setting $v_{e e}^{\lambda}=\lambda / r_{12}$, which leads to Eq. (12). If one is able to compute the exact $f_{c}^{\lambda}$, the
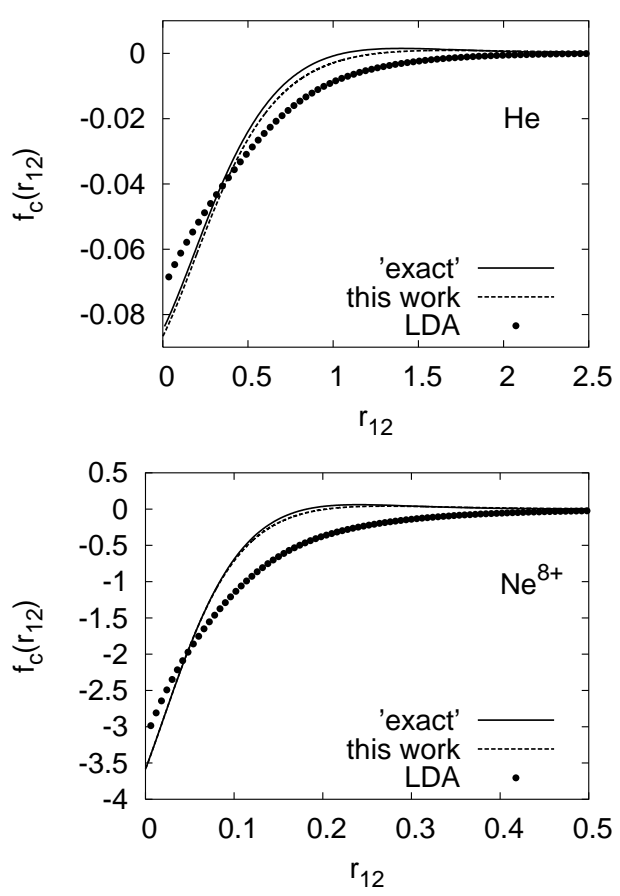

FIG. 2: The correlated part of the spherically- and systemaveraged pair density, $f_{c}\left(r_{12}\right)=f\left(r_{12}\right)-f_{\mathrm{KS}}\left(r_{12}\right)$. Our results for $\mathrm{He}$ and $\mathrm{Ne}^{8+}$ are compared with the "exact" ones and with the LDA result (the hole for the uniform electron gas is taken from Ref. [46]).

resulting $E_{c}$ from Eq. (21) is independent of the choice of $v_{e e}^{\lambda}$. However, when approximations are made some "paths" can give much better results than others [6]. As we shall see, this is the case with the present approach.

We build an Overhauser-like potential for interaction $v_{e e}^{\lambda}$ (to be added to $v_{\text {eff }}^{(0)}$ ) as

$$
v_{\mathrm{eff}}^{O v, \lambda}\left(r_{12} ; \bar{r}_{s}\right)=v_{e e}^{\lambda}\left(r_{12}\right)-\int_{|\mathbf{r}| \leq \bar{r}_{s}} \bar{n} v_{e e}^{\lambda}\left(\left|\mathbf{r}-\mathbf{r}_{12}\right|\right) d \mathbf{r} .
$$

That is, the average density $\bar{n}$ of Eq. (17) (and thus the average $\bar{r}_{s}$ ) is kept fixed to mimic the fact that the one-electron density does not change along the adiabatic connection. The modified interaction $v_{e e}^{\lambda}$ is screened by a sphere of radius $\bar{r}_{s}$ and of positive uniform charge of density $\bar{n}$ that attracts the electrons with the same modified interaction. This attractive background approximates the effect of the external potential $V^{\lambda}$ on $f$.

\section{A. Linear adiabatic connection}

If we choose $v_{e e}^{\lambda}=\lambda / r_{12}$ we simply obtain $v_{\text {eff }}^{O v, \lambda}\left(r_{12} ; \bar{r}_{s}\right)=\lambda v_{\text {eff }}^{O v}\left(r_{12} ; \bar{r}_{s}\right)$, where $v_{\text {eff }}^{O v}\left(r_{12} ; \bar{r}_{s}\right)$ is given by Eq. (14).

The results for $\left\langle V_{e e}^{\lambda}\right\rangle-\left\langle V_{e e}\right\rangle_{K S}$ for $\mathrm{He}$ and $\mathrm{Ne}^{8+}$ are shown in Fig. 4 and are compared with the "exact" ones of Ref. [42]. The correlation energy $E_{c}$ can be 

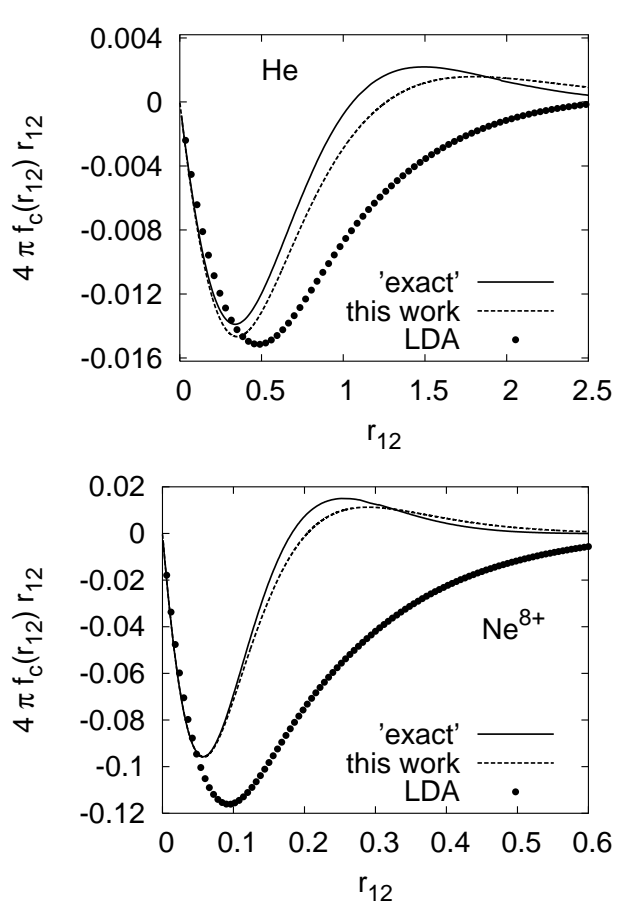

FIG. 3: The real space analysis of the correlation part of the expectation value of $V_{e e}$ : the area under each curve gives $\left\langle V_{e e}\right\rangle-\left\langle V_{e e}\right\rangle_{K S}$ (see also Eq. (4) and Fig. 2). Our results for $\mathrm{He}$ and $\mathrm{Ne}^{8+}$ are compared with the "exact" ones and with the LDA result (the hole for the uniform electron gas is taken from Ref. [46]).

calculated as the area under each curve. We obtain $E_{c}=-0.052$ Hartree for $\mathrm{He}$ and $E_{c}=-0.053$ Hartree for $\mathrm{Ne}^{8+}$, to be compared with the corresponding 'exact' results, -0.042 and -0.045 , respectively.

\section{B. A nonlinear adiabatic connection}

As shown by Figs. 13 and Table【 the Overhauser-like potential gives accurate results for the short-range part of $f_{c}\left(r_{12}\right)$. We can thus expect to obtain better correlation energies from the adiabatic connection formalism if we choose a modified interaction $v_{e e}^{\lambda}$ that is able to separate long-range and short-range contributions, like the "erf" interaction [6, 35, 36, 37]

$$
v_{e e}^{\lambda}\left(r_{12}\right)=\frac{\operatorname{erf}\left(\lambda r_{12}\right)}{r_{12}}
$$

With this choice, Eq. (21) becomes

$$
E_{c}[n]=\int_{0}^{\infty} d \lambda \int_{0}^{\infty} d r_{12} 4 \pi r_{12}^{2} f_{c}^{\lambda}\left(r_{12}\right) \frac{2}{\sqrt{\pi}} e^{-\lambda^{2} r_{12}^{2}}
$$

For large $\lambda$, when we are approaching the physical system, the gaussian factor $e^{-\lambda^{2} r_{12}^{2}}$ in Eq. (24) quenches the long-range contribution of $f_{c}^{\lambda}$ to the energy integrand. At the KS end of the adiabatic connection, when $\lambda \rightarrow 0$, the
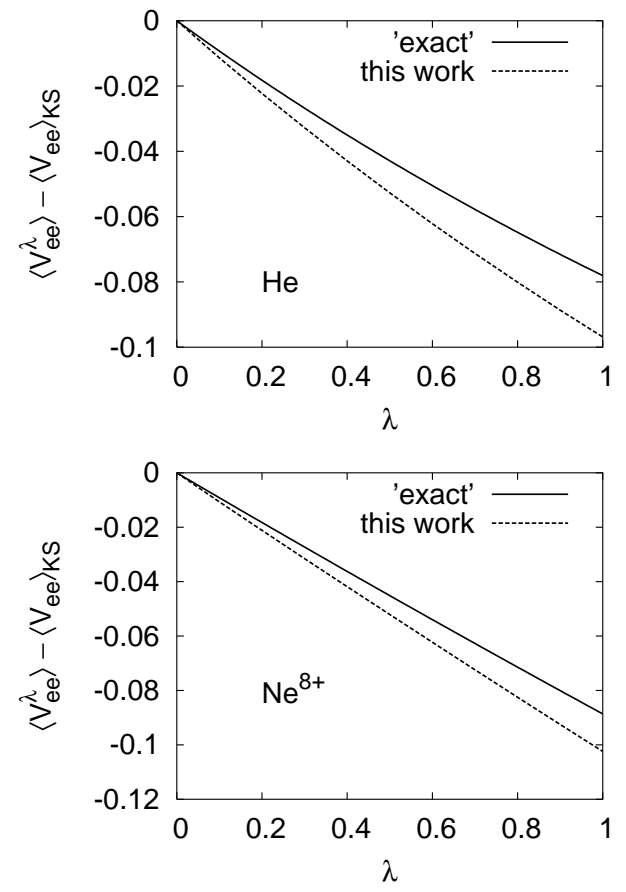

FIG. 4: Correlation part of $\left\langle V_{e e}^{\lambda}\right\rangle$ along the linear adiabatic connection for $\mathrm{He}$ and $\mathrm{Ne}^{8+}$. Our results are compared with the "exact" ones of Ref. [42]. The area under each curve gives the correlation energy $E_{c}$ of standard Kohn-Sham theory.

interaction, and thus $f_{c}^{\lambda}$, become small, so that the contribution to $E_{c}$ coming from $\lambda$-values for which the longrange part of $f_{c}^{\lambda}$ is not quenched is moderate. Moreover, the function $f_{c}^{\lambda}$ is correctly normalized to zero so that for $\lambda \rightarrow 0$, not only is $f_{c}^{\lambda}$ small, but also the integral itself vanishes. In the linear adiabatic connection of Eq. (12), instead, the long-range part of $f_{c}^{\lambda}$ plays an important role in the energy integrand at all $\lambda$. Indeed, with this nonlinear adiabatic connection we obtain $E_{c}=-0.0405$ Hartree for $\mathrm{He}$ and $E_{c}=-0.0413$ for $\mathrm{Ne}^{8+}$, much closer to the "exact" values with respect to the results from the linear adiabatic connection.

The technical details of this calculation are as follows. The potential $v_{\text {eff }}^{O v, \lambda}\left(r_{12} ; \bar{r}_{s}\right)$ of Eq. (22) can be computed analytically, and is reported in Appendix \$. We thus obtained, via Eqs. (6)-(7), $d E_{c}^{\lambda} / d \lambda=$ $\int d \mathbf{r}_{12} f_{c}^{\lambda}\left(r_{12}\right) \frac{2}{\sqrt{\pi}} e^{-\lambda^{2} r_{12}^{2}}$ for 23 values of $\lambda$ between 0 and 20 for $\mathrm{He}$, and between 0 and 100 for $\mathrm{Ne}^{8+}$. We then fitted our results with the derivative of the following functional form

$$
E_{c}^{\lambda}=-\frac{a_{1} x^{6}+a_{2} x^{8}+a_{3} x^{10}}{\left(1+b^{2} x^{2}\right)^{5}}, \quad x=\frac{\lambda}{Z},
$$

that has exact asymptotic behaviors for small and large $\lambda$ [37]. (We have numerical evidence that our results fulfill such exact behaviors.) In Fig. 5 we report our numerical values for $d E_{c}^{\lambda} / d \lambda$, together with the derivative of the fitting function of Eq. (25). The parameters and 

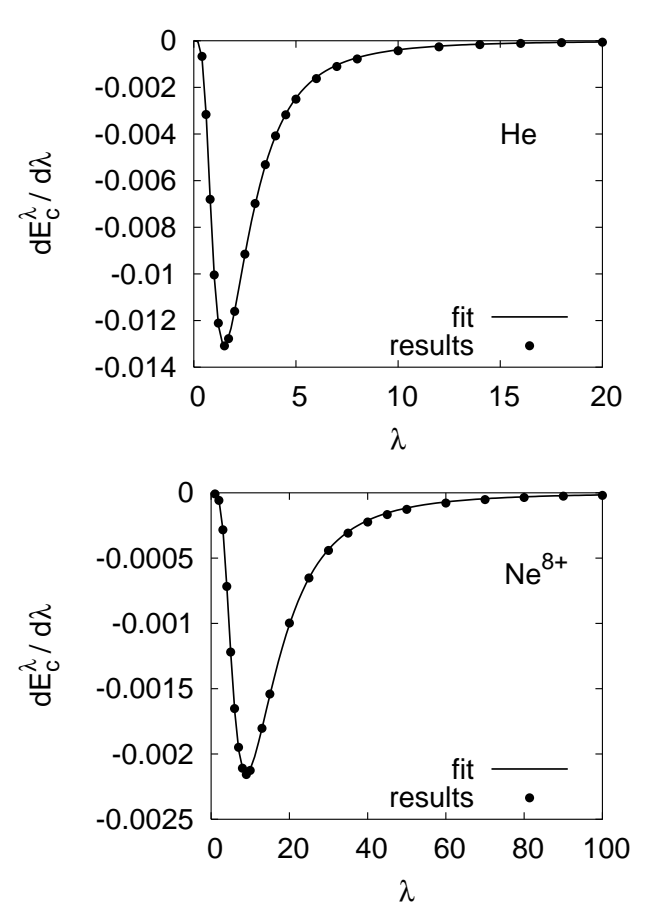

FIG. 5: The derivative $d E_{c}^{\lambda} / d \lambda$ along the nonlinear adiabatic connection defined by Eqs. (23)-(24) for $\mathrm{He}$ and $\mathrm{Ne}^{8+}$. Our results are compared with the derivative of the fitting function of Eq. (25). The area under each curve from zero to $\infty$ gives the correlation energy $E_{c}$ of standard Kohn-Sham theory.

the r.m.s of residuals are reported in Table [II The KS correlation energy $E_{c}[n]$ is then given by $a_{3} / b^{10}$.

The accuracy of our results with the "erf" adiabatic connection is of particular interest for the method of Refs. 6, 35, 36, 37], which combines multideterminantal wavefunctions (configuration interaction, CI) with density functional theory ("CI+DFT"). In such approach, instead of the KS system, one choses a reference system of partially interacting particles, usually with the potential of Eq. (23). This model system is treated with a multideterminantal wavefunction, in a CI fashion, that allows to treat near-degeneracy effects. The remaining part of the energy is calculated via a density functional, that needs to be approximated. The larger $\lambda$, the larger is the energy fraction treated with the CI calculation, and thus the larger is the computational cost. The correlation energy functional that needs to be approximated is $6,35,36,37$

$$
\bar{E}_{c}^{\lambda}[n] \equiv E_{c}[n]-E_{c}^{\lambda}[n],
$$

and can be rewritten as

$$
\bar{E}_{c}^{\lambda}[n]=\int_{\lambda}^{\infty} d \lambda^{\prime} \int_{0}^{\infty} d r_{12} 4 \pi r_{12}^{2} f_{c}^{\lambda^{\prime}}\left(r_{12}\right) \frac{2}{\sqrt{\pi}} e^{-\lambda^{\prime 2} r_{12}^{2}}
$$

Thus, only the short-range part of $f_{c}^{\lambda}$ contributes to the functional $\bar{E}_{c}^{\lambda}[n]$, and we expect to get accurate results with the present approach. Indeed, this is the case, as

\begin{tabular}{lccccc}
\hline \hline & $a_{1}$ & $a_{2}$ & $a_{3}$ & $b$ & r.m.s. \\
\hline $\mathrm{He}$ & 1.2047 & 2.3253 & 2.7788 & 1.5263 & $4 \cdot 10^{-5}$ \\
$\mathrm{Ne}^{8+}$ & 0.3983 & 0.4711 & 0.4026 & 1.2557 & $9 \cdot 10^{-6}$ \\
\hline \hline
\end{tabular}

TABLE II: Optimal fit parameters and r.m.s of the residuals for the derivative of Eq. (25), that parametrizes our results for $d E_{c}^{\lambda} / d \lambda$ along the nonlinear adiabatic connection defined by Eqs. (23)- 24). See also Fig. 5
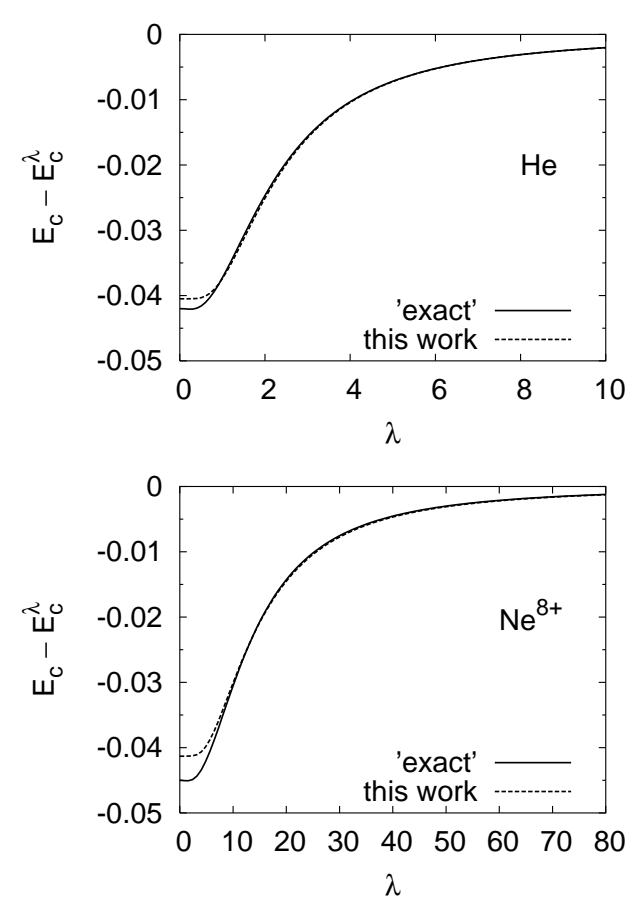

FIG. 6: A nonlinear adiabatic connection that separates longand short-range effects: difference between the correlation energy $E_{c}$ of the physical system (with full interaction $1 / r$ ) and the correlation energy $E_{c}^{\lambda}$ of the system with partial interaction $\operatorname{erf}(\lambda r) / r$, for $\mathrm{He}$ and $\mathrm{Ne}^{8+}$. Our results are compared with the "exact" ones of Ref. [36].

shown in Fig. 6] where we compare our results as a function of $\lambda$ with the "exact" ones of Ref. [36]. The error is less than $0.5 \mathrm{mHartree}$ for $\lambda \gtrsim 1 / \bar{r}_{s}$, that is a very reasonable choice for the value of $\lambda$ to be used in the CI+DFT method of Refs. [6, 35, 36, 37].

\section{CONCLUSIONS AND PERSPECTIVES}

In this work we have started to explore the possibility of solving simple radial equations to generate realistic spherically- and system-averaged electronic pair densities $f\left(r_{12}\right)$ for nonuniform systems. With a simple approximation for the unknown effective electron-electron interaction that appears in our formalism, we have obtained, for two-electron atoms, results that are in fair agreement with those coming from accurate variational wavefunc- 
tions (Figs. 10 3 and Table प). We have then extended our approach along a nonlinear adiabatic connection and obtained Kohn-Sham correlation energies whose error is less than 4 mHartrees, and short-range-only correlation energies whose accuracy is one order of magnitude better (Fig. 6).

In Sec. II we have introduced a general formalism for many-electron systems that will be further tested in future work. So far we can say that this formalism, combined with simple physical approximations, works very well for two completely different systems: the uniform electron gas [23, 24, 25] and the He series. We think that this fact makes the method promising.

To fully develop the approach described in this paper, many steps have to be performed. First of all, the KS part of the effective e-e potential, $v_{\text {eff }}^{(0)}\left(r_{12}\right)$ of Eq. (18), should be also approximated, to make the extension to many-electron systems practical. The correlation part of the effective $e$-e potential can be improved, in analogy with the recent developments for the uniform electrongas case 24, 25]. It should then be possible to construct a self-consistent scheme (OEP-like) that combines the Kohn-Sham equations with the correlation energy functional arising from our approach [Eqs. (6)-(7) at different coupling strengths $\lambda$, plus Eq. (12) or Eq. (24)]. With respect to traditional DFT calculations, this combined scheme would have the advantage of yielding not only the ground-state one-electron density $n(\mathbf{r})$ and energy $E$, but also the spherically- and system-averaged pair density $f\left(r_{12}\right)$, thus allowing to calculate expectation values of two-body operators that only depend on the electronelectron distance. The combination of our approach with the CI+DFT method of Refs. [6, 35, 36, 37] could be also implemented and, in view of the results of Fig. [6 it is even more promising. We are presently working in all of these main directions.

\section{Acknowledgments}

We thank C. Umrigar for the wavefunctions of Ref. [40], J. Toulouse for the results of Ref. [36], E.K.U. Gross, W. Kohn, M. Polini, G. Vignale and P. Ziesche for encouraging discussions, V. Sahni for useful hints, and J.K. Percus for helpful suggestions. This research was supported by a Marie Curie Intra-European Fellowships within the 6th European Community Framework Programme (contract number MEIF-CT-2003-500026).

\section{APPENDIX A: OVERHAUSER-LIKE POTENTIAL FOR THE ERF INTERACTION}

The evaluation of Eq. (22) with the interaction $v_{e e}^{\lambda}=$ $\operatorname{erf}\left(\lambda r_{12}\right) / r_{12}$ gives

$$
v_{\mathrm{eff}}^{O v, \lambda}\left(r_{12} ; \bar{r}_{s}\right)=\frac{u(s, \mu)}{\bar{r}_{s}},
$$

where $s=r_{12} / \bar{r}_{s}, \mu=\lambda \bar{r}_{s}$, and

$$
\begin{aligned}
& u(s, \mu)=\frac{\operatorname{erf}(\mu s)}{s}-\frac{1}{8 \sqrt{\pi} s \mu^{3}}\left\{2\left[1+\left(-2+s+s^{2}\right) \mu^{2}+e^{-4 s \mu^{2}}\left(-1+\left(2+s-s^{2}\right) \mu^{2}\right)\right] e^{-(1-s)^{2} \mu^{2}}-\right. \\
& \left.\sqrt{\pi} \mu\left[3 s+2(1-s)^{2}(2+s) \mu^{2}\right] \operatorname{erf}[\mu(1-s)]+\sqrt{\pi} \mu\left[-3 s+2(2-s)(1+s)^{2} \mu^{2}\right] \operatorname{erf}[\mu(1+s)]\right\} .
\end{aligned}
$$

[1] W. Kohn, Rev. Mod. Phys. 71, 1253 (1999).

[2] A.E. Mattsson, Science 298, 759 (2002).

[3] C. Fiolhais, F. Nogueira, and M. Marques (eds.), A Primer in Density Functional Theory (Springer-Verlag, Berlin, 2003).

[4] W. Kohn and L.J. Sham, Phys. Rev. 140, A1133 (1965).

[5] J.P. Perdew and K. Schmidt, in Density Functional Theory and Its Applications to Materials, edited by V. VanDoren et al. (AIP, NY, 2001), and references therein.

[6] A. Savin, F. Colonna, and R. Pollet, Int. J. Quantum Chem. 93, 166 (2003), and references therein.

[7] V. Sahni, Quantal Density Functional Theory (SpringerVerlag, Berlin, 2004).

[8] J.P. Perdew, K. Burke, and M. Ernzerhof, Phys. Rev. Lett. 77, 3865 (1996); ibid. 78, 1396 (1997); A.D. Becke,
Phys. Rev. A 38, 3098 (1988); J. Chem. Phys. 84, 4524 (1986); C. Lee, W. Yang, and R.G. Parr, Phys. Rev. B 37, 785 (1988); J.P. Perdew, J.A. Chevary, S.H. Vosko, K.A. Jackson, M.R. Pederson, D.J. Singh, and C. Fiolhais, Phys. Rev. B. 46, 6671 (1992); ibid. 48, 4978 (1993).

[9] J. Tao, J.P. Perdew, V.N. Staroverov, and G.E. Scuseria, Phys. Rev. Lett. 91, 146401 (2003).

[10] J.P. Perdew, A. Ruzsinszky, J. Tao, V.N. Staroverov, G.E. Scuseria, and G.I. Csonka, J. Chem. Phys., to appear.

[11] M. Seidl, J.P. Perdew, and S. Kurth, Phys. Rev. Lett. 84, 5070 (2000).

[12] see, e.g., S. Kümmel and J.P. Perdew, Phys. Rev. B 68, 035103 (2003); W. Yang and Q. Wu, Phys. Rev. Lett. 89, 143002 (2002); R. J. Magyar, A. Fleszar, and E. K. U. 
Gross, Phys. Rev. B 69, 045111 (2004); M. Grüning, O. V. Gritsenko, and E. J. Baerends, J. Chem. Phys. 118, 7183 (2003).

[13] C.A. Coulson and A.H. Neilson, Proc. Phys. Soc. London 78, 831 (1961).

[14] J. Cioslowski, B.B. Stefanov, A. Tan, and C.J. Umrigar, J. Chem. Phys. 103, 6093 (1995).

[15] J. Cioslowski and G. Liu, J. Chem. Phys. 109, 8225 (1998).

[16] E. Valderrama, J.M. Ugalde, and R.J. Boyd, in Manyelectron densities and reduced density matrices, edited by J. Cioslowski (Kluwer Academic/Plenum Publishers, New York, 2000).

[17] E.R. Davidson, Reduced Density Matrices in Quantum Chemistry (Academic Press, New York, 1976).

[18] A.J. Coleman and V.I. Yukalov, Reduced Density Matrices: Coulson's Challenge (Springer-Verlag, New York, 2000).

[19] see, e.g., G. Mazzone, F. Sacchetti, and V. Contini, Phys. Rev. B 28, 1772 (1983); C. Petrillo and F. Sacchetti, ibid. 51, 4755 (1995).

[20] A. K. Rajagopal, J. C. Kimball, and M. Banerjee, Phys. Rev. B 18, 2339 (1978); X.-Y. Pan and V. Sahni, J. Chem. Phys. 119, 7083 (2003).

[21] The difference in kinetic energy can be obtained from the adiabatic connection formula that can be viewed as a generalization of the above argument, and is treated in Sec. V

[22] A.W. Overhauser, Can. J. Phys. 73, 683 (1995).

[23] P. Gori-Giorgi and J.P. Perdew, Phys. Rev. B 64, 155102 (2001).

[24] B. Davoudi, M. Polini, R. Asgari, and M.P. Tosi, Phys. Rev. B 66, 075110 (2002).

[25] M. Corona, P. Gori-Giorgi, and J.P. Perdew, Phys. Rev. B 69, 045108 (2004); I. Nagy, R. Diez Muiño, J.I. Juaristi, and P.M. Echenique, Phys. Rev. B 69, 233105 (2004).

[26] P. Ziesche, Phys. Lett. A 195, 213 (1994); M. Levy and P. Ziesche, J. Chem. Phys. 115, 9110 (2001).

[27] A. Gonis, T.G. Schulthess, J. van Ek, and P.E.A. Turchi, Phys. Rev. Lett. 77, 2981 (1996); A. Gonis, T.G. Schulthess, P.E.A. Turchi, and J. van Ek, Phys. Rev. B 56, 9335 (1997).

[28] A. Nagy, Phys. Rev. A 66, 022505 (2002).

[29] F. Furche, Phys. Rev. A 70, 022514 (2004).

[30] J.M. Soler, Phys. Rev. B 69, 195101 (2004).

[31] M. Levy, Proc. Natl. Acad. Sci. U.S.A. 76, 6062 (1979).

[32] E. Lieb, Int. J. Quantum Chem. 24, 243 (1983).

[33] J. Harris and R. Jones, J. Phys. F 4, 1170 (1974); D.C.
Langreth and J.P. Perdew, Solid State Commun. 17, 1425 (1975); O. Gunnarsson and B.I. Lundqvist, Phys. Rev. B 13, 4274 (1976).

[34] W. Yang, J. Chem. Phys. 109, 10107 (1998).

[35] A. Savin, in Recent Developments and Applications of Modern Density Functional Theory, edited by J.M. Seminario (Elsevier, Amsterdam, 1996); T. Leininger, H. Stoll, H.-J. Werner, and A. Savin, Chem. Phys. Lett. 275, 151 (1997); R. Pollet, A. Savin, T. Leininger, and H. Stoll, J. Chem. Phys. 116, 1250 (2002).

[36] R. Pollet, F. Colonna, T. Leininger, H. Stoll, H.-J. Werner, and A. Savin, Int. J. Quantum Chem. 91, 84 (2003); J. Toulouse, private communication.

[37] J. Toulouse, F. Colonna, and A. Savin, Phys. Rev. A 70, 062505 (2004).

[38] B. Davoudi, R. Asgari, M. Polini, and M.P. Tosi, Phys. Rev. B 68, 155112 (2003); R. Asgari, B. Davoudi, and M.P. Tosi, Solid State Comm. 131, 301 (2004).

[39] R. Asgari, B. Davoudi, M. Polini, and M.P. Tosi, in preparation.

[40] D.E. Freund, B.D. Huxtable, and J.D. Morgan III, Phys. Rev. A 29, 980 (1984). We used an improved version (provided to us by C. Umrigar) of the accurate variational wavefunctions described in this work to obtain oneelectron densities $n(\mathbf{r})$ and functions $f\left(r_{12}\right)$. See also C.J. Umrigar and X. Gonze, Phys. Rev. A 50, 3827 (1994), and Ref. 14].

[41] P. Ziesche, Phys. Rev. B 67, 233102 (2003); P. Ziesche, K. Pernal, and F. Tasnádi, Phys. Status Solidi B 239, 185 (2003). In these papers interesting exact properties of the "Overhauser geminals" are derived, using the interacting momentum distribution to define the geminal occupancy. Notice however that the uniform-electron gas equivalent of our Eqs. (6)-(7) employ the non-interacting momentum distribution, as it has been done in Refs. [23, 24, 25].

[42] F. Colonna and A. Savin, J. Chem. Phys. 110, 2828 (1999).

[43] Q. Zhao, R. C. Morrison, and R. G. Parr, Phys. Rev. A 50, 2138 (1994); R. van Leeuwen and E. J. Baerends Phys. Rev. A 49, 2421 (1994).

[44] J.P. Perdew, A. Savin, and K. Burke, Phys. Rev. A, 51, 4531 (1995); K. Burke, J.P. Perdew, and M. Ernzerhof, J. Chem. Phys. 109, 3760 (1998); E. Valderrama and J.M. Ugalde, Int. J. Quantum Chem. 86, 40 (2002).

[45] X. Fradera, M. Duran, E. Valderrama, and J.M. Ugalde, Phys. Rev. A 62, 034502 (2000).

[46] P. Gori-Giorgi and J.P. Perdew, Phys. Rev. B 66, 165118 (2002). 